\title{
A. ЭЭНСААР
}

удК $551.5 ! 1$

\section{ЧИСЛЕННЫЙ МЕТОД ПАРАМЕТРИЗАЦИИ ПОГРАНИЧНОГО СЛОЯ АТМОСФЕРЫ}

\author{
(Представил Х. Абен)
}

Взаимодействие атмосферы с поверхностью Земли приводит к возникновению турбулентного пограничного слоя, в пределах которого в уравнениях движения и переноса тепла и влаги необходимо учитывать наличие вертикальных турбулентных потоков [ $\left.{ }^{1}\right]$. Основными характеристиками взаимодействия между атмосферой и подстилающей поверхностью являются вертикальные турбулентные потоки тепла $H=c_{p} \bar{w}^{\prime} T^{\prime}$ и водяного пара $E=\overline{w^{\prime} q^{\prime}}$, а также турбулентное напряжение трения $\vec{\tau}$, составляющие которого $\tau_{x}=-\overline{\varrho w^{\prime} u^{\prime}}$ и $\tau_{y}=-\overline{w^{\prime} v^{\prime}}$. Здесь принята декартова система координат. Оси $x$ и $y$ расположены в горизонтальной плоскости, ось $z$ - по вертикали. Обозначения следующие: $T, \varrho, c_{p}-$ абсолютная температура воздуха, его плотность и теплоемкость при посто. янном давлении; $q$ - удельная влажность; $u, v$, w- составляющие скорости ветра соответственно по направлению осей $x, y, z$. Штрихом обозначены пульсации, а черта сверху означает осреднение. Вертикальный турбулентный поток импульса равен турбулентному напряжению трения $\tau=|\vec{\tau}|$. В приземном слое направление ветра и тангенциального напряжения трения совпадают.

Правильное описание и моделирование турбулентных потоков необходимо для решения целого ряда прикладных задач. Так, например, при решении задач распространения в воздухе загрязняющих веществ одним из основных этапов является моделирование реального поля ветра. При этом ключевым является учет взаимодействия атмосферы с подстилающей поверхностью, т. е. установление связей между вертикальными потоками количества движения, тепла и влаги на уровне подстилающей поверхности с внешними по отношению к пограничному слою метеорологическими параметрами.

Для решения упомянутой задачи можно применить теорию подобия атмосферного пограничного слоя (АПС) $\left[{ }^{2}\right]$. Согласно этой" теории, характеристики турбулентного режима АПС выражаются через универсальные функции от двух переменных - интегрального числа Ричардсона $R i$ и числа Россби $R o$, определяемых следующим образом

$$
\begin{aligned}
& R i=\left[\beta\left(\Theta_{h}-\Theta_{0}\right)+0,61 g\left(q_{h}-q_{0}\right)\right] /|f| G, \\
& R o=G /|f| z_{0},
\end{aligned}
$$

где $\Theta$ - потенциальная температура $\left(\Theta_{h}\right.$ и $q_{h}-$ значения на верхней границе АПС, $\Theta_{0}$ и $q_{0}-$ на уровне шероховатости); $\beta$ - параметр плавучести; $f$ - параметр Кориолиса; $G$ - модуль скорости геострофического ветра; g - ускорение свободного падения; $z_{0}-$ шероховатость поверхности. 


$$
\begin{aligned}
\tau & =\mathrm{Q}^{2} G^{2} \Phi_{1}(R i, R o) \\
\alpha & =\Phi_{2}(R i, R o), \\
H & =-c_{p} \mathrm{Q} G\left(\Theta_{h}-\Theta_{0}\right) \Phi_{3}(R i, R o), \\
E & =-{ }_{\mathrm{Q}} G\left(q_{h}-q_{0}\right) \Phi_{3}(R i, R o) .
\end{aligned}
$$

Здесь $\tau, H, E$ - приповерхностные вертикальные турбулентные потоки импульса, тепла и влаги соответственнс; $\alpha$ - угол поворота ветра в АПС, т. е. угол между направлением вектора $\left(\tau_{x}, \tau_{y}\right)$ тангенциального напряжения трения у Земли и вектора $(u, v)$ горизонтальной скорости ветра в свободной атмосфере.

В $\left[{ }^{3}\right]$ приведены уравнения, аппроксимирующие соответствующие универсальные функции в виде

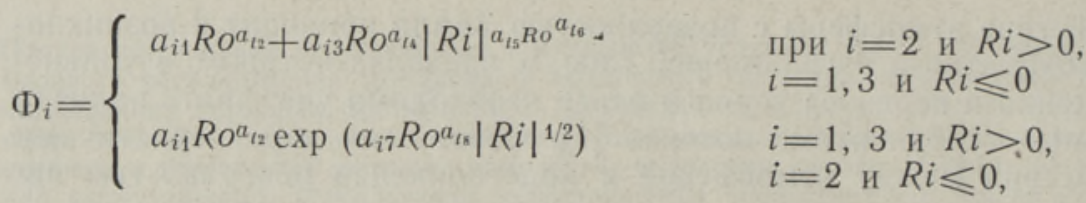

где $a_{i j}-$ коэффициенты, первый индекс которых совпадает с номером функции $\Phi_{i}$.

Теория подобия АПС, строго говоря, описывает стационарный и однородный по горизонтали пограничный слой. Однако при нахождении зависимостей (7) использован экспериментальный материал, имеющий явный суточный ход. Если характерные горизонтальные масштабы процессов достаточно велики (как минимум на порядок больше толщины пограничного слоя), то теория подобия АПС должна соблюдаться и при нестационарных условиях над неоднородной местностью. Главной причинсй нестационарности метеорологического режима в АПС являются периодические колебания потока коротковолновой солнечной радиации, падающего на подстилающую поверхность.

Для практического применения теории подобия в целях прогноза требуется знать температуру подстилающей поверхности, которую нельзя считать постоянной в течение суток. В данной работе приведем метод решения этой задачи.

Предположим, что характеристики геострофического потока известны из глобального прогноза. Кроме того, считаем, что известны и теплофизические свойства подстилающей поверхности, ее шероховатость и среднесуточная температура. Для определения температуры поверхности земли используем уравнение баланса тепла

$$
P_{0}+H+a_{E} L E=I(1-A)-F,
$$

где $P_{0}$ - теплопередача через поверхность почвы; $L-$ скрытая теплота испарения; $a_{E}$ - безразмерный коэффициент, позволяющий учесть тот факт, что в разных точках подстилающей поверхности вследствие ее неоднородности на исппарение тратится неодинаковое количество тепла $\left[{ }^{4}\right] ; I-$ коротковолновая солнечная радиация; $A-$ альбедо; $F-$ эффективное излучение.

Отметим, что в ряде случаев разница между $\Theta_{0}$ и средней потенциальной температурой $\Theta_{s}$ в точках непосредственного контакта воздуха с подстилающей поверхностью может достигать довольно больших значений. При решении уравнения (8) используем полуэмпирическую параметризацию для вязкого подслоя [ $\left.{ }^{1}\right]$

$$
\Theta_{0}-\Theta_{s}=0,13 T_{\star}\left(z_{0} u_{\star} / v\right)^{0,45},
$$


где $T_{*}=-H / x c_{p} \varrho u_{*}-$ масштаб температуры; $x$ - постоянная Кармана; $u_{*}=\sqrt{\tau / \varrho}-$ скорость трения; $v-$ кинематический коэффициент вязкости воздуха.

Удельную влажность вычисляем по уравнению

$$
q=r q_{k}(T),
$$

где $r$ - относительная влажность, предполагаемая известной функция от времени; $q_{k}-$ насыщающая удельная влажность.

Эффективное излучение представим в виде

$$
F=\sigma_{s} T_{0}^{4},
$$

где $\sigma_{s}-$ постоянная Стефана-Больцмана, умноженная на коэффициснт, учитывающий влияние влажности воздуха, облачности и серости почвы.

Будем искать приближенное решение уравнения (8). Для этого перейдем от непрерывного аргумента к дискретному. Введем временной индекс $j$. Шаг по времени $\Delta t$ выбираем так, чтобы обеспечивалась малость изменений температуры в течение одного шага. Разложим выражения для $H, E, F$ и $T_{s}$ в ряд Тейлора, где ограничимся только первыми членами. Для $H$, например, имеем

$$
H^{j}=H^{j-1}+\left(\frac{\partial H}{\partial \Theta_{0}}\right)^{j-1}\left(\Theta_{0}^{j}-\Theta_{0}^{j-1}\right) .
$$

Уравнения (1)-(11) позволяют получить аналитические выражения для необходимых производных. При этом надо учитывать, что $\lim _{R i \rightarrow 0}\left|\frac{\partial \Phi_{3}}{\partial R i}\right|=\infty$. Такой ход производной в окрестности $R i=0$ с физической точки зрения не оправдывается и связан с конкретным выбором аппроксимирующих уравнений $(7)$. В данной области $\Phi_{3}$ по величине изменяется мало (рис. 1). Следовательно, в этой области можно вместо $\frac{\partial \Phi_{3}}{\partial R i}$ применять $\frac{\Delta \Phi_{3}}{\Delta R i}$.

【ля решения уравнения (8) необходимо еще найти поток тепла в почву. Гаспределение температуры в почве описывается уравнением

$$
\frac{\partial T}{\partial t}=K_{s} \frac{\partial^{2} T}{\partial z^{2}} \quad(z \leqslant 0)
$$

где $t-$ время; $K_{s}-$ коэффициент температуропроводности почвы. Принимаем, что

$$
T=\bar{T}+\delta,
$$

где $\delta$ - отклонение температуры почвы от среднесуточного значения $\bar{T}=$ const. Уравнение (13) рсшается при условии $\delta=0$, если $z \rightarrow-\infty$. Допустим, что шаг по времени $\Delta t$ настолько мал, что можно без существенной погрешности на каждом интервале $\Delta t$ интерполировать $\delta_{0}$ линейной функцией $t$. Тогда согласно $\left[{ }^{5,6}\right]$, поток тепла в почву вычисляется следующим образом

$$
\begin{gathered}
P_{0}=B \sum_{n=0}^{\infty} k_{n} \delta_{0}^{j-n}, \\
B=2 \lambda_{s} /\left(K_{s} \pi \Delta t\right)^{1 / 2} ; \\
k_{0}=1 ; \quad k_{n}=(n+1)^{1 / 2}-2 n^{1 / 2}+(n-1)^{1 / 2} \text { при } n \geqslant 1 .
\end{gathered}
$$

Здесь $\lambda_{s}-$ коэффициент теплопроводности почвы. 
$\log$

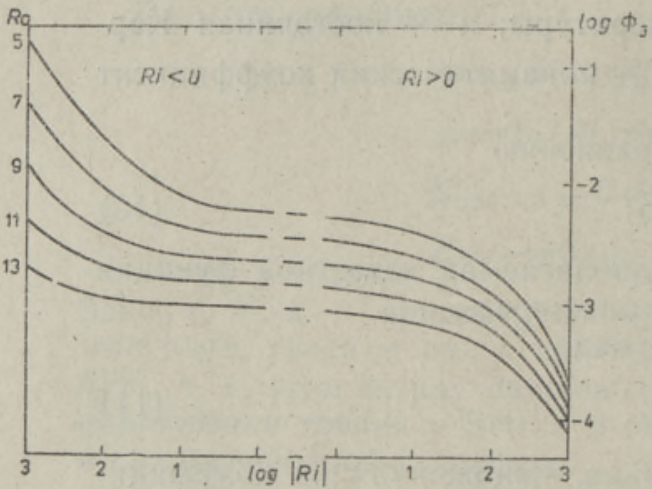

Рис. 1. Зависимость универсальной функции $\Phi_{3}$ (вычислена по (7)) от интегрального числа Ричардсона $R i$ и числа Россби Ro. Значеняя $\log R o$ указаны в начале кривых.
Используя уравнения (14), и результаты разложения в ряд Тейлора, можем решить уравнение (8)

$$
\begin{gathered}
\Theta_{0}^{j=}=\Theta_{0}^{j-1}+D\left[I^{j}(1-A)-B\left(0,414 \delta_{0}^{j-1}+\sum_{n=2}^{\infty} k_{n} \delta_{0}^{j-n}\right)-\right. \\
\left.-H^{j-1}-a_{E} L E^{j-1}-\sigma_{s}\left(T_{0}^{j-1}\right)^{4}\right]
\end{gathered}
$$

Здесь

$$
D=\left[B \mu+\left(\frac{\partial H}{\partial \Theta_{0}}\right)^{j-1}+a_{E} L\left(\frac{\partial E}{\partial \Theta_{0}}\right)^{j-1}+4 \sigma_{s} \varepsilon\left(T_{0}^{j-1}\right)^{3}\right]^{-1}
$$

$\mu=\left(\frac{\partial T_{s}}{\partial \Theta_{0}}\right)^{j-1} ; \varepsilon=\left(\frac{p}{p_{0}}\right)^{\frac{R}{c_{p}}} ; \quad p-$ давление воздуха;

$p_{0}=1000$ гПа - нормальное давление; $R$ - газовая постоянная воздуха.

Уравнение (15) представляет собой т. н. решение без начальных условий. При использовании уравнения (16) надо знать температуру поверхности земли при всех моментах времени $j^{\prime}=j-n(n \geqslant 1)$, предшествующих моменту $j$. Однако для достижения необходимой для практических целей точности можем учитывать в бесконечной сумме лишь нессколько первых членов.

Описанный метод реализован в виде подпрограммы на ФОРТРАНе.

Ниже приводим результаты расчета на один модельный день. Для параметризации радиационных потоков использованы уравнения Альбрехта и Брента $\left[{ }^{7}\right]$

$$
\begin{aligned}
& I=\left(a_{0} \sin h_{c}-b_{0}\left(\sin h_{c}\right)^{1 / 2}\right)\left(1-c_{1} n\right), \\
& \sin h_{c}=\sin \varphi \sin \psi+\cos \varphi \cos \psi \cos \tau_{c}, \\
& F=\sigma f_{s} T_{0}^{4}\left(a_{2}-b_{2}(e)^{1 / 2}\right)\left(1-c_{2} n\right) .
\end{aligned}
$$

Здесь $h_{c}-$ зенитный угол Солнца; $\varphi-$ широта места; $\psi-$ склонение Солнца; $n-$ облачность; $\tau_{c}-$ часовой угол; $f_{s}-$ коэффициент серости почвы; $e$ - упругость водяного пара; $a_{0}, b_{\curvearrowleft}, a_{2}, b_{2}, c_{1}, c_{2}-$ эмпирические константы. Численный эксперимент (рис. 2) проводился при следующих значениях входных параметров: $a_{0}=1291 \mathrm{Bт} / \mathrm{m}^{2} ; b_{0}=223 \mathrm{BT} / \mathrm{m}^{2}$; $n=0 ; \quad \varphi=1,037 ; \quad f_{s}=0,9 ; \quad a_{2}=0,39 ; \quad b_{2}=0,077 ; \quad \lambda_{s}=1,84 \cdot 10^{6} \quad \mathrm{BT} /(\mathrm{M} \cdot \mathrm{K}) ;$ $K_{s}=1,25 \cdot 10^{-6} \quad \mathrm{~m}^{2} / \mathrm{c} ; \quad a_{E}=0,20 ; \quad G=8,4 \mathrm{~m} / \mathrm{c} ; \quad \Theta_{h}=291,5 \mathrm{~K} ; \quad \bar{T}=$ $=291,0 \mathrm{~K} ; z_{0}=0,05 \mathrm{м} ; \Delta t=1200 \mathrm{c} ; \quad p=1015 \mathrm{r \Pi а} ; A=0,2 ; \psi=0,409$. В качестве начальных данных принято, что $\delta_{0}^{j-n}=0$ при $n \geqslant 1$. Ввиду периодичности радиационных потоков тепла решение $\Theta_{0}$ быстро пере- 


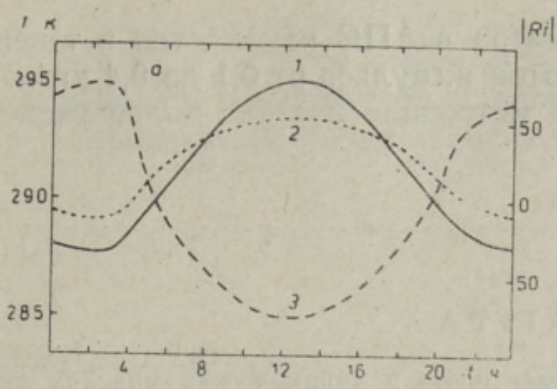

Рис. 2. Суточный ход характеристик режима турбулентности в АПС. Результаты численного экспернмента.

a: 1 - потенциальная температура поверхности, 2 - потенциальная температура воздуха иа уровне шероховатости, 3 - интегральное число Ричардсона;

б: 1 - радиационный баланс, 2 - поток скрытого тепла, 3 - турбулентный поток тепла, 4 - поток тепла в почву;

в: 1 - приповерхностный поток нмпульса, 2 - угол поворота ветра в АПС.
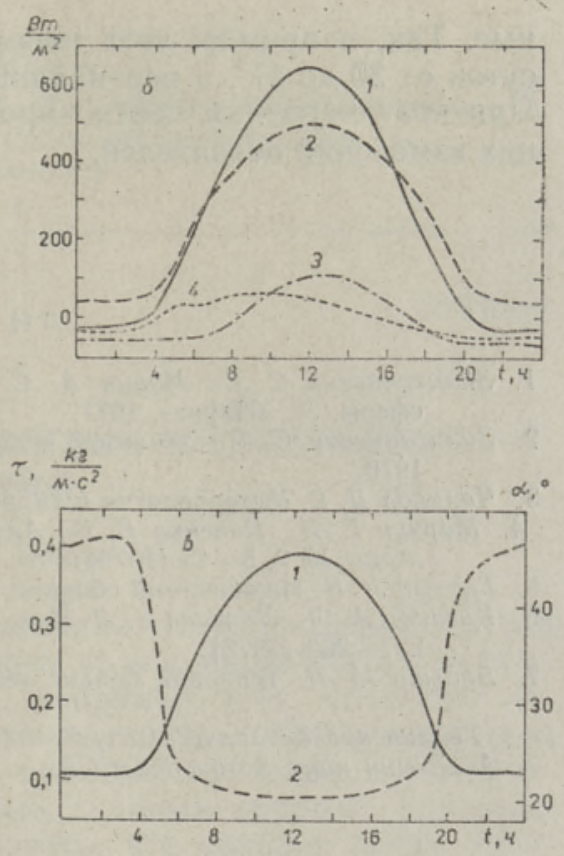

ходит на периодический режим. Если при определении $\Theta_{0}$ считать допустимой абсолютную погрешность $0,01 \mathrm{~K}$, то время приспособления $\mathrm{K}$ периодическому режиму в рассматриваемом случае не превышает 7 ч.

Суточный ход вычисленных величин (рис. 2) качественно согласуется с результатами, полученными другими авторами $\left[{ }^{2,7}\right]$. Количественное соответствие связано с точностью определения параметров и начальных данных. Кроме того, несоответствие может возникать при резкой нестационарности (в условиях восхода и захода Солнца).

Численными экспериментами установлено, что при $\Delta t=1200$ с учет члена $\frac{\partial \Phi_{3}}{\partial R i}$ является несущественным. Разница между вычисленными температурами почвы составляет примерно $0,03 \mathrm{~K}$. В этом случае можем принимать $R i^{j} \approx R i^{j-1}$, т. е. вычислять $\Phi_{3}$ по результатам счета предыдущего шага. Решение уравнения баланса тепла можно тогда представить в виде

$$
\Theta_{0}^{j}=\left(\tilde{\Theta}_{0}+D \Theta_{h}\right) /(1+D)
$$

где

$$
\begin{gathered}
D=c_{p \varrho} G \Phi_{3}^{j-1} W \\
W=\left[a_{E} L\left(\frac{\partial E}{\partial \Theta_{0}}\right)^{j-1}+4 \sigma_{s} \varepsilon\left(T_{0}^{j-1}\right)^{3}+B \mu\right]^{-1} \\
\tilde{\Theta}_{0}=\Theta_{0}^{j-1}+W\left[I^{j}(1-A)-B\left(0,414 \delta_{0}^{j-1}+\sum_{n=2}^{\infty} k_{n} \delta_{0}^{j-n}\right)-\right. \\
\left.-a_{E} L E^{j-1}-\sigma_{s}\left(T_{0}^{j-1}\right)^{4}\right] .
\end{gathered}
$$

Уравнения (16) или (20) и (1)-(7) позволяют учитывать в численных моделях изменения турбулентного режима пограничного слоя атмосферы в течение суток. Из рис. 2 видно, что эти изменения существен- 
ные. Так, например, угол поворота ветра в АПС изменяется в течение суток от 20 до $47^{\circ}$, а вертикальный поток импульса от 0,1 до $0,4 \mathrm{kr} / \mathrm{m} \cdot \mathrm{c}^{2}$. При краткосрочном прогнозировании метеорологических полей учет таких изменений обязателен.

\section{ЛИТЕРАТУРА}

1. Зилитинкевич С. С., Монин А. С. Турбулентность в динамических моделях атмосферы. Л., «Наука», 1971.

2. Зилитинкевич C. С. Динамика пограничного слоя атмосферы. Л., Гидрометсоиздат, 1970.

3. Чаликов Д. В. Метеорология и гидрология, № 8, 12-15 (1977).

4. Марчук Г. И., Пененко В. В., Алоян А. Е., Лазриев Г. Л. Метеорология и гидрология, № 8, 5-15 (1979).

5. Гутман Л. Н. Инженерный сборник, 15, 99-136 (1953).

6. Казаков А. Л., Лазриев $Г$. Л. Изв. АН СССР. Физика атмосферы и океана, 14, № 3, $257-265$ (1978)

7. Будыко М. И. Тепловой баланс земной поверхности. Л., Гидрометеоиздат, 1956.

Таллинский ботанический сад Академии наук Эстонской ССР

\section{Поступила в редакцию} $19 /$ V 1983

\section{A. EENSAAR}

\section{ATMOSFÄARI PIIRIKIHI PARAMEETRIMISE NUMBERMEETOD}

On csitatud meetod maapinnalähedaste vertikaalsete turbulentsete voogude arvutamiseks. Turbulentsusrežiim atmosfääri piirikihis sőltub integraalsest Richardsoni arvust ja Rossby arvust. Maapinna temperatuur on leitud soojusbilansi vorrrandist. Arvutused näitavad, et ööpäeva kestel muutub turbulentsusrežiim oluliselt.

\section{A. EENSAAR}

\section{A NUMERICAL METHOD FOR THE ATMOSPHERIC BOUNDARY LAYER PARAMETRIZATION}

A numerical model to esetimate the turbulent fluxes near the ground surface is presented. The interaction between the air mass and the underlying surface is described bv the similarity theory of the atmospheric boundary layer (ABL). The surface fluxes of momentum, heat and moisture as well as the angle between the surface wind and the isobars, are described as functions of Richardson number and Rossby number $(3-6)$. The surface temperature is determined according to the heat budget equation $(16,20)$. In Fig. 2 the diurnal variation of some calculated values characterizing ABL-s turbulence, are presented. 